\title{
Pulse solutions of the cubic-quintic complex Ginzburg-Landau equation in the case of normal dispersion
}

\author{
J. M. Soto-Crespo \\ Instituto de Óptica, Consejo Superior de Investigaciones Científicas, Serrano 121, 28006 Madrid, Spain \\ N. N. Akhmediev and V. V. Afanasjev \\ Optical Sciences Centre, The Australian National University, Canberra 0200 ACT, Australia \\ S. Wabnitz \\ Laboratoire de Physique, Université de Bourgogne, Avenue Alain Savary, 21004 Dijon, France
}

(Received 11 November 1996)

\begin{abstract}
Time-localized solitary wave solutions of the one-dimensional complex Ginzburg-Landau equation (CGLE) are analyzed for the case of normal group-velocity dispersion. Exact soliton solutions are found for both the cubic and the quintic CGLE. The stability of these solutions is investigated numerically. The regions in the parameter space in which stable pulselike solutions of the quintic CGLE exist are numerically determined. These regions contain subspaces where analytical solutions may be found. An investigation of the role of group-velocity dispersion changes in magnitude and sign on the spectral and temporal characteristics of the stable pulse solutions is also carried out. [S1063-651X(97)10504-9]

PACS number(s): 42.65.Tg, 42.25.Bs, 42.55.Wd
\end{abstract}

\section{INTRODUCTION}

The emergence of stable spatiotemporal patterns in a variety of physical situations may be modeled through the wellknown complex Ginzburg-Landau equation (CGLE). In the simplest situation where a single transverse (or temporal) coordinate is retained in the analysis, the CGLE reads [1]

$$
\begin{gathered}
i \psi_{z}+\frac{D}{2} \psi_{t t}+|\psi|^{2} \psi=i \delta \psi+i \epsilon|\psi|^{2} \psi+i \beta \psi_{t t}+i \mu|\psi|^{4} \psi \\
-\nu|\psi|^{4} \psi
\end{gathered}
$$

The CGLE applies, for example, to describing self-phase modulation of light in a dispersive medium (e.g., an optical fiber). In this case, $t$ is a retarded time, $z$ is the propagation distance, $\delta, \beta, \epsilon, \mu$, and $\nu$ are real constants (we do not require them to be small), $\psi$ is a complex field and $D= \pm 1$ is the dispersion coefficient.

Many nonequilibrium phenomena, such as the generation of spatiotemporal dissipative structures in lasers [2-4], binary fluid convection [5,6], phase transitions [7], and soliton propagation in optical fiber systems with linear and nonlinear gain and spectral filtering (such as communication links with lumped fast saturable absorbers [8-15] or fiber lasers with additive-pulse mode-locking or nonlinear polarization rotation [1,16-27]), may all be described by the CGLE.

For the specific case of the optical fiber mentioned above, the physical meaning of these quantities is the following: $\psi$ is the complex envelope of the electric field, $\delta$ is the linear gain, $\beta$ describes spectral filtering $(\beta>0), \epsilon$ accounts for nonlinear gain-absorption processes, $\mu$ represents a higherorder correction to the nonlinear amplification or absorption, and $\nu$ is a possible higher-order correction term to the intensity-dependent refractive index.
Equation (1) is written in such a way that if the right-hand side of it is set to zero, then one is simply left with the nonlinear Schrödinger equation (NLSE). In the case of anomalous dispersion $(D=+1)$, the solitary wave solutions of the CGLE and their stability have been analyzed in previous papers $[28,29]$. As is well known, in the anomalous dispersion regime bright soliton solutions of the NLSE equation exist. In this case, for relatively small values of the various terms on the right-hand side of Eq. (1), the solitary wave solutions of the CGLE with $D=+1$ are close to the usual NLSE solitons. As a consequence, in the anomalous dispersion regime it is possible to study the main properties of the solitonlike solutions of Eq. (1) by applying the welldeveloped soliton perturbation theory of the NLSE [30,31]. This approach, however, cannot be used with normal dispersion [i.e., $D=-1$ in Eq. (1)], since in this case no solution of the NLSE in the form of a bright pulse exists. The question then arises about the existence of any localized or bright pulse solution of the CGLE. If so, given that soliton perturbation theory is not applicable, it is then necessary to characterize the bright pulse solutions of Eq. (1) with $D=-1$ by means of direct analytical or numerical methods.

As a matter of fact, many physical systems described by the CGLE do indeed lead to the observation of stable pulselike solutions even in the case of normal group-velocity dispersion. For example, in soliton fiber lasers it has been observed that ultrashort (i.e., of the order of $100 \mathrm{fs}$ ) pulses are formed and emitted also with a normal average cavity dispersion $[20,21,32,33]$. Not only the pulses in the normal dispersion regime are as stable as in the case of anomalous dispersion: the observations also demonstrated that, when the magnitude of the normal dispersion coefficient grows larger, a useful increase of the pulse energy results.

It is well known that, in the case of normal dispersion without gain and loss, the balance of dispersion and nonlinearity can only support dark solitons. However, if, in addi- 
tion, one includes the balance of linear and nonlinear (cubic) gain and loss, then bright solitary waves are possible even in the normal dispersion regime: in fact, the analytical expression for these chirped hyperbolic-secant profile pulses was known for quite a long time [34-36]. However, it is quite easy to show that such solitary waves are unstable, unless the quintic nonlinear loss (or gain saturation) term is also included. It appears that a comprehensive study of the characteristics and stability of the bright solitary wave solutions of Eq. (1) has not been carried out. Thus we dedicate this work to the above interesting task.

As was just mentioned, the case of the cubic CGLE was extensively studied (see, e.g., [34,37-39]) and its general solution, i.e., a pulse with fixed amplitude, is well known. Quite recently, new types of solutions (namely, stable solutions with arbitrary amplitude) were found numerically [40] and analytically [28]. It turns out that such solutions also exist for normal dispersion. However, as we shall discuss below, in this case such solutions are unstable.

The quintic CGLE was considered in a number of publications using numerical simulations, perturbative analysis, and analytic solutions. Perturbative analysis of the solitons of the quintic CGLE in the NLSE limit (i.e., for the anomalous dispersion regime) was developed in [41-43]. The existence of solitonlike solutions of the quintic CGLE in the case of subcritical bifurcations $(\epsilon>0)$ was also numerically determined $[43,44]$. More recently, the regions in the parameter space at which stable pulselike solutions exist were found for the case of anomalous dispersion in Ref. [29]. A qualitative analysis of the transformation of the regions of existence of the pulselike solutions, when the coefficients on the righthand side change from zero to infinity, was done by Hakim, Jakobsen, and Pomeau [42]. An analytic approach, based on the reduction of Eq. (1) to a three-variable dynamical system, which allows one to obtain exact solutions for the quintic equation, was developed by van Saarloos and Honhenberg $[45,46]$. However, no explicit solutions were given.

The mathematical treatment of the exact solutions of the quintic CGLE, using Painlevé analysis and symbolic computations, has appeared in the recent work by Marcq, Chaté, and Conte [47]. The general approach, used in [47], is the reduction of the differential equation to a purely algebraic problem. Here we are using a simpler approach that was developed in [28] for the anomalous dispersion case. We first proceed by generalizing that approach to deal with arbitrary values of the dispersion coefficient. Then we analyze the stability of the various solutions, and finally seek for the regions in the parameter space where stable pulselike solutions exist. In this work we restrict our attention to the most relevant specific case for the optical applications; that is, the time-localized pulselike solutions of the CGLE.

The rest of the paper is organized as follows. The general ansatz and the analytical procedure are described in Sec. II. Exact solutions of the cubic CGLE are described in Sec. III, where we also study their stability. The quintic CGLE solutions are obtained and analyzed in Sec. IV. These analytical treatments are done for an arbitrary value of $D$. Stable solutions are then numerically found in Sec. V, where we determine the regions in the parameter space where they exist. In Sec. V, the numerical results deal with the case $D=-1$. We then lift this restriction in Sec. VI, where we analyze the effect of changing both the magnitude and sign of the dispersion $D$ on the spectral and temporal characteristics of the stable pulse solutions of the CGLE, for a specific choice of the other parameters. We then discuss all the previous results in Sec. VII. Finally, we summarize our conclusions in Sec. VIII.

\section{ANALYTICAL PROCEDURE}

To find analytic solutions, we follow the procedure outlined in [28], which we now generalize for any value of the parameter $D$. We first consider the stationary solutions of Eq. (1) with zero transverse velocity. Traveling solutions for $\beta=0$ are considered in a special section. Hence we look for a solution of the form

$$
\psi(t, z)=a(t) \exp [i \phi(t)] \exp (-i \omega z),
$$

where $\omega$ is a real constant, and $a$ and $\phi$ are real functions of $t$. Assuming that

$$
\phi(t)=d \ln [a(t)],
$$

where $d$ is the phase modulation parameter, known as chirp in nonlinear optics, and, after some cumbersome transformations (see Ref. [28]), one obtains three algebraic equations relating the different parameters, and an ordinary differential equation for $a(t)$, namely,

$$
\begin{gathered}
\nu\left(4 d+2 D \beta d^{2}-6 D \beta\right)+\mu\left(8 D \beta d-d^{2}+3\right)=0, \\
\omega=-\frac{\delta\left(1-d^{2}+4 D \beta d\right)}{2\left(d-D \beta+D \beta d^{2}\right)}, \\
d=d_{ \pm}=\frac{(3+6 D \epsilon \beta) \pm \sqrt{(3+6 D \epsilon \beta)^{2}+8(\epsilon-2 D \beta)^{2}}}{2(\epsilon-2 D \beta)}, \\
\frac{a^{\prime 2}}{a^{2}}+\frac{2 \nu}{8 \beta d-D d^{2}+3 D} a^{4}+\frac{2(2 \beta-D \epsilon)}{3 d\left(1+4 \beta^{2}\right)} a^{2} \\
-\frac{\delta}{\beta d^{2}+D d-\beta}=0 .
\end{gathered}
$$

Equation (6) is an important result, it shows that $d$ can be found in terms of $\beta$ and $\epsilon$ only, and therefore its expression is the same for both the cubic and the quintic CGLE. The coefficient in front of $a^{4}$ in Eq. (7) can also be written in another way, taking into account Eq. (4),

$$
\frac{2 \nu}{8 \beta d-D d^{2}+3 D}=\frac{\mu}{3 \beta-2 D d-\beta d^{2}} .
$$

In what follows, we consider the solitons of the cubic and the quintic CGLE separately. In each section, we derive the analytical solution and then look for special cases and singularities.

Equation (3) is, obviously, a restriction imposed on $\phi(t)$, because the phase modulation could have a more general functional dependence on $t$. However, this restriction allows us to find some families of solutions in analytical form. For the cubic case, our ansatz covers all pulselike so- 
lutions. In the quintic case, the solutions reported in this paper are only those which can be represented in forms (2) and (3).

\section{SOLITONS OF THE CUBIC CGL EQUATION}

\section{A. Solitons with fixed amplitude}

First, we consider the cubic CGLE, that is Eq. (1) with $\nu=\mu=0$. Then Eq. (7) has the solution

$$
a(t)=B C \operatorname{sech}(B t)
$$

where

$$
C=\left(\frac{3 d\left(1+4 \beta^{2}\right)}{2(2 \beta-D \epsilon)}\right)^{1 / 2}, \quad B=\left(\frac{\delta}{D d-\beta+\beta d^{2}}\right)^{1 / 2}
$$

and $d$ is given by Eq. (6), after choosing the plus (minus) sign in front of the square root if $D$ is negative (positive). The second value of $d$ leads to an unphysical solution (see Ref. [28] for details), as the expression under the square root for $C$ becomes negative. Solution (9) has been found by Pereira and Stenflo [34] (see also [37-39]). An important feature of the solution (9) is that its amplitude and width depend uniquely on the parameters of the equation. This is a common property of the solutions of nonconservative systems. In other words, Eq. (9) is the solution with fixed amplitude.

Note that solution (9) depends on four parameters, $\delta, \beta$, $\epsilon$, and $D$. Parameter $\delta$ appears only in the expression for $B$ [see Eq. (10)], so a variation of $\delta$ leads to a rescaling of the soliton amplitude only. As in this paper we are interested primarily in the normal dispersion case, we fix the value of the dispersion $D=-1$. So the further study is conducted mainly on the $(\beta, \epsilon)$ plane.

To find the range of existence of solution (9), note that, on the $(\beta, \epsilon)$ plane, the denominator in the expression for $B$ is positive below the curve $S$ given by

$$
\epsilon_{S}=\beta \frac{3 \sqrt{1+4 \beta^{2}}-D}{4+18 \beta^{2}},
$$

and negative above it [see Fig. 1(a)]. Hence, for solution (9) to exist, the value $\delta$ must be positive below the curve $S$ and negative above it. As this solution exists almost everywhere on the $(\beta, \epsilon)$ plane, we call it the general solution. The curve $S$ itself is the line where this solution becomes singular, i.e., its amplitude $B C$ tends to infinity, while the width $1 / B$ vanishes.

We have found numerically, using the technique developed in [48] (see also Ref. [29] for more precise details), that all these solutions are unstable. Figure 1(b) shows the growth rate of the most unstable eigenperturbation associated to each stationary solution for $D=-1$. The curves on the left are for $\epsilon=0.4$ with $\beta$ in the interval $[0,1]$, and those on the right are for $\beta=0.1$ with $\epsilon$ in the interval $[0,1]$. The solid lines are for $\delta=-0.1$, and the dashed lines for $\delta=-0.2$. This figure shows the following: (i) the perturbation growth
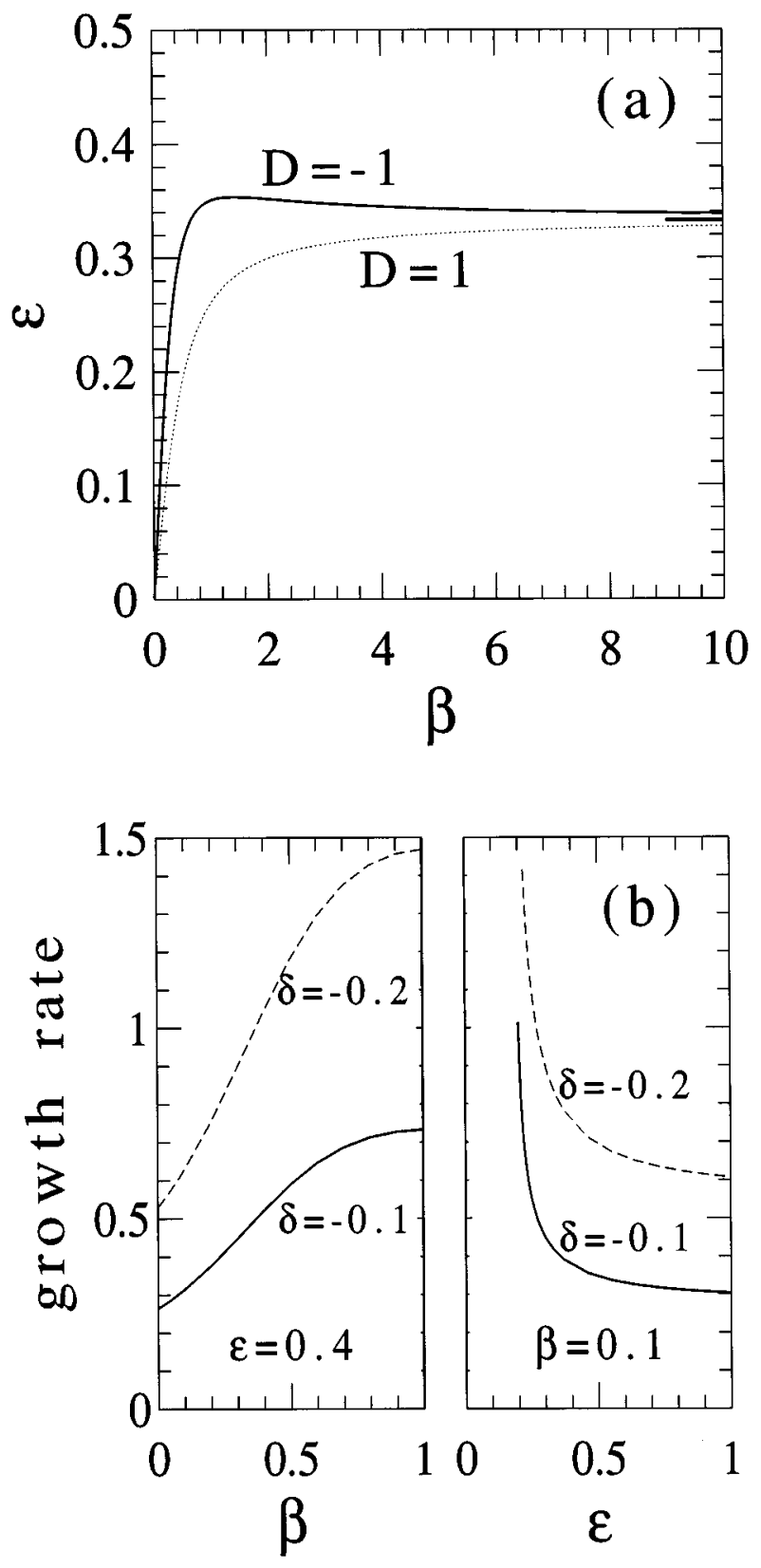

FIG. 1. (a) Line (11) (line $S$ ) on the plane $(\epsilon, \beta)$ where the solutions with fixed amplitudes (9) and (24) become singular, and where the classes of special solutions with arbitrary amplitude (12) and (28) exist. This plot applies for both the cubic and quintic cases. The corresponding one for the case of anomalous dispersion is also shown by the dotted line for comparison. Above line $S, \delta$ must be positive for solution (9) to exist, and negative below it. The dotted line is line $S$ for $D=+1$ [28]. (b) The growth rate of the predominant perturbation eigenmode associated with the solution of fixed amplitude of the cubic CGLE (9). The curves on the left are for $\epsilon=0.4$ and $\beta$ in the interval $[0,1]$, and those on the right for $\beta=0.1$ and $\epsilon$ in the interval $[0,1]$. Solid lines are for $\delta=-0.1$, and dashed ones for $\delta=-0.2$.

rate increases as either $|\delta|$ or $\beta$ increases, (ii) it decreases as $\epsilon$ increases (but the decline saturates for $\epsilon>0.5$ ), and (iii) it never goes to zero.

On the other hand, for positive linear amplification below 

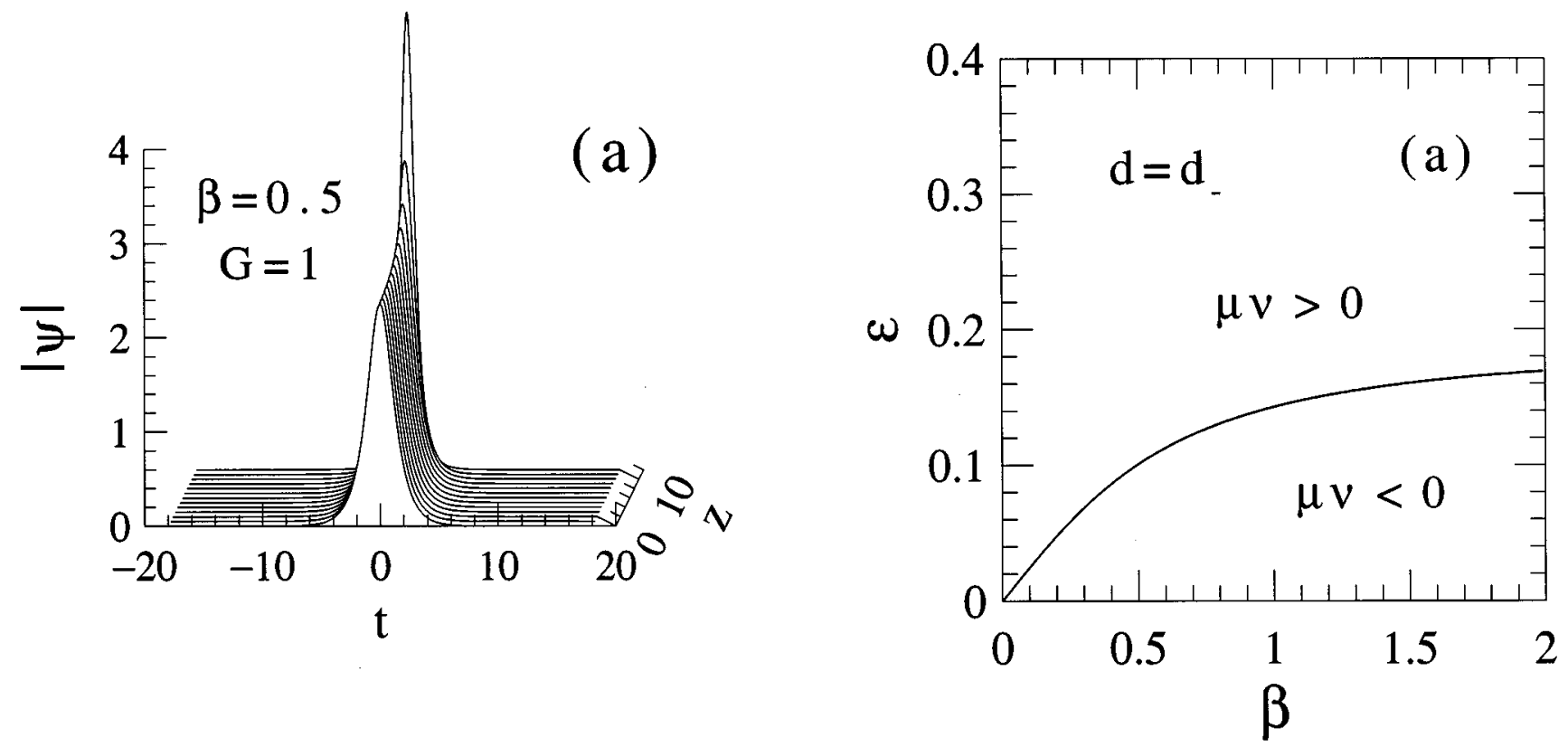

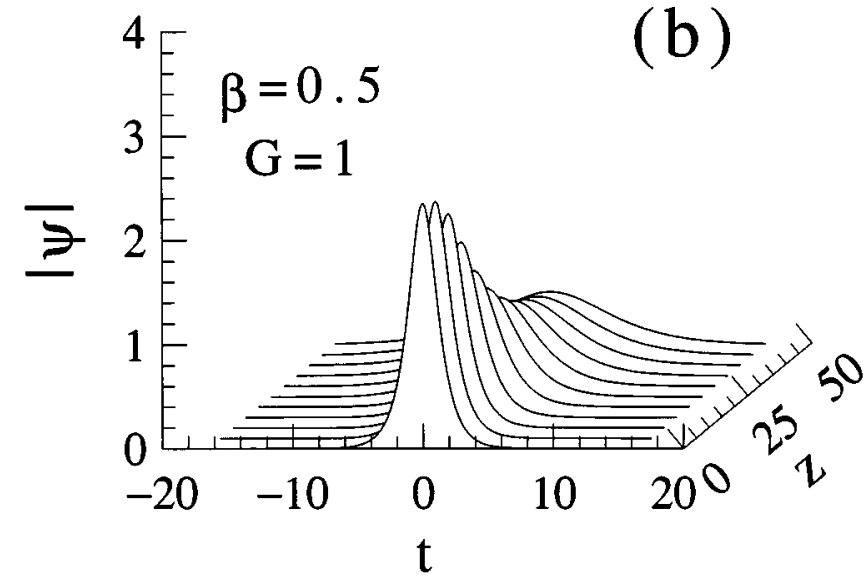

FIG. 2. The two possible types of evolution of a stationary solution of the cubic CGLE, as given by Eq. (16), for $G=1$ and $\beta=0.5$.

the line $S(\delta>0)$, the background state $(\psi=0)$ becomes unstable. Accordingly, we numerically found that the corresponding perturbation growth rate is exactly equal to $\delta$, except in the vicinity of the line $S$, where it grows without limit. If the initial conditions are close to the exact solution (9) and $\delta \ll 1$, this instability develops slowly and the soliton can propagate for distances up to $z_{0} \sim \delta^{-1}$. Beyond that, radiation waves, growing linearly from the noise, become appreciable and can distort the soliton itself. However, in other problems $\delta$ can be large, so $z_{0}$ is small. The general conclusion is that either the soliton itself, or the background state, is unstable at each point in the plane $(\epsilon, \beta)$. This means that the total solution is always unstable.

\section{B. Solution with arbitrary amplitude}

It is easy to see that solution (9) does not exist on line $S$ [Eq. (11)]. However, if we also impose the condition $\delta=0$, a solution, valid only on line (11), can be found:

$$
a(t)=G F \operatorname{sech}(G t),
$$

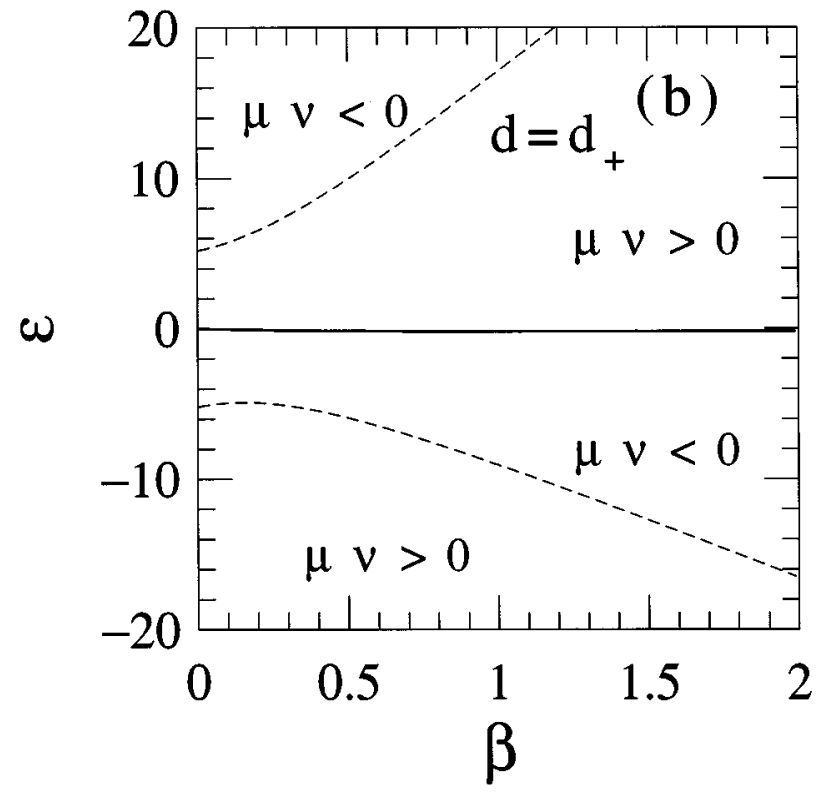

FIG. 3. The relation between the parameters $\mu$ and $\nu$ on the semiplane $(\epsilon, \beta)$, for which the quintic CGL has analytic solutions. (a) The case of negative sign in Eq. (6). (b) The case of positive sign in Eq. (6). The continuous lines represent the points $(\beta, \epsilon)$ where $\mu=0$, and the dashed lines those where $\nu=0$.

where $G$ is an arbitrary positive parameter, and $d, \omega$, and $F$ are given by

$$
d=\frac{\sqrt{1+4 \beta^{2}}-D}{2 \beta},
$$

$$
\omega=-\frac{\left(1+4 \beta^{2}\right)\left(\sqrt{1+4 \beta^{2}}-D\right)}{4 \beta^{2}} G^{2}=-d \frac{1+4 \beta^{2}}{2 \beta} G^{2}
$$




$$
\begin{aligned}
F & =\left(\frac{d \sqrt{1+4 \beta^{2}}}{2 \epsilon}\right)^{1 / 2} \\
& =\left[\frac{\left(2+9 \beta^{2}\right) \sqrt{1+4 \beta^{2}}\left(\sqrt{1+4 \beta^{2}}-D\right)}{2 \beta^{2}\left(3 \sqrt{1+4 \beta^{2}}-D\right)}\right]^{1 / 2} .
\end{aligned}
$$

Solution (12) represents the arbitrary-amplitude soliton.

The reason for the existence of the arbitrary-amplitude solutions is that, when $\delta=0$, the cubic CGLE becomes invariant relative to the scaling transformation $\psi \rightarrow G \psi$, $t \rightarrow G t, z \rightarrow G^{2} z$. Hence, if we know a particular solution of this equation, the whole family can be generated using this transformation. Note that all parameters of solution (12) (except $G$ ) and the coefficient $\epsilon$ are expressed in terms of $\beta$.

We have found, from numerical simulations, that contrarily to what happens in the anomalous dispersion regime [28], the class of arbitrary-amplitude solutions for $D=-1$ is unstable relative to small perturbations at any point of the line $S$. Figure 2 shows the two possible ways of evolution of these solutions. As initial conditions, we used

$$
\begin{aligned}
& \text { (a) } \psi(z=0, t)=1.001 A(t), \\
& \text { (b) } \psi(z=0, t)=0.999 A(t),
\end{aligned}
$$

where $A(t)$ is the corresponding stationary solution (i.e., $A(t)=a(t) \exp [d \ln (a(t))]$, with $a(t)$ given by Eq. (12)) for the following coefficients $G=1, D=-1, \delta=0$, and $\beta=0.1 \quad(\Rightarrow \epsilon=0.308)$. The solution either grows without limit [see Fig. 2(a)] or decays, diminishing in amplitude and increasing its width [see Fig. 2(b)].

\section{SOLITONS OF THE QUINTIC CGL EQUATION}

\section{A. Relation between coefficients}

The soliton solutions of the quintic CGLE exist for a wide range of values of the coefficients $\beta, \epsilon, \mu$, and $\nu$. Ansatz (3) is the condition that restricts this range by imposing the relation [Eq. (4)] on them. Using Eq. (6), this relation can be rewritten as a linear equation in $d$ :

$$
\begin{aligned}
\nu\left[\frac{12 \epsilon \beta^{2}+4 \epsilon-2 D \beta}{\epsilon-2 D \beta} d-2 D \beta\right] \\
+\mu\left[\frac{2 D \epsilon \beta-16 \beta^{2}-3}{\epsilon-2 D \beta} d+1\right]=0 .
\end{aligned}
$$

We can also eliminate $d$ completely from Eqs. (4) and (6) to obtain the following relation for $\epsilon$ :

$$
\epsilon=\frac{4 D \beta \mu^{2}+30 \mu \nu+120 \beta^{2} \mu \nu+4 D \beta \nu^{2} \pm 3 U}{-\mu^{2}+12 D \beta \mu \nu+32 \nu^{2}+108 \beta^{2} \nu^{2}},
$$

where

$$
U=\sqrt{(\mu-2 D \beta \nu)^{2}\left(3 \mu^{2}+16 \beta^{2} \mu^{2}+4 D \beta \mu \nu+4 \nu^{2}+12 \beta^{2} \nu^{2}\right)} .
$$

This expression gives the relation between the coefficients in an explicit form. In contrast to the cubic equation, the general solution exists for both signs in expression (6) for $d$.

Now we consider the zero of $\mu$ and $\nu$ in the $(\beta, \epsilon)$ plane. $\mu$ has to be zero on the lines (solid ones in Fig. 3 for $D=-1)$

$$
\epsilon=D \beta \frac{1 \pm 3 \sqrt{1+3 \beta^{2}}}{8+27 \beta^{2}}
$$

for $d=d_{ \pm}$, respectively, and $\nu$ becomes zero on the two lines [the dashed lines in Fig. 3(b) for $D=-1$ ] defined by

$$
\epsilon= \pm 3 \sqrt{16 \beta^{2}+3}-4 D \beta
$$

both for $d=d_{+}$. The value of the product $\mu \nu$ changes sign on these lines (see Fig. 3). These conclusions can be made more specific when we consider more particular regions of existence of these solutions.

In what follows, we consider solutions which exist when at least one of the coefficients $\mu$ or $\nu$ is nonzero, and express the solutions in terms of $\beta, \epsilon$, and $\nu$. Using Eq. (8), the solutions can alternatively be expressed in terms of $\beta, \epsilon$, and $\mu$.

\section{B. Solutions with fixed amplitude}

By using the substitution $f=a^{2}$ we can rewrite Eq. (7) in the form

$$
\begin{aligned}
& \frac{f^{\prime 2}}{f^{2}}+\frac{8 \nu}{8 \beta d-D d^{2}+3 D} f^{2}+\frac{8(2 \beta-D \epsilon)}{3 d\left(1+4 \beta^{2}\right)} f-\frac{4 \delta}{D d-\beta+\beta d^{2}} \\
& \quad=0 .
\end{aligned}
$$

This is an elliptic-type differential equation. Bounded solitonlike solutions exist only if

$$
\frac{4 \delta}{D d-\beta+\beta d^{2}}>0 \text {. }
$$

The positive solution of Eq. (22) is [49]

$$
f(t)=\frac{2 f_{1} f_{2}}{\left(f_{1}+f_{2}\right)-\left(f_{1}-f_{2}\right) \cosh \left(2 \alpha \sqrt{f_{1}\left|f_{2}\right|} t\right)},
$$

where

$$
\alpha=\left(\left|\frac{2 \nu}{8 \beta d-D d^{2}+3 D}\right|\right)^{1 / 2}=\left(\left|\frac{\mu}{3 \beta-2 D d-\beta d^{2}}\right|\right)^{1 / 2},
$$

and $f_{1}$ and $f_{2}$ are given by 


$$
f_{1,2}=\frac{-(2 \beta-D \epsilon) \pm\left((2 \beta-D \epsilon)^{2}+\frac{18 \delta d^{2} \nu\left(1+4 \beta^{2}\right)^{2}}{\left(8 \beta d-D d^{2}+3 D\right)\left(D d-\beta+\beta d^{2}\right)}\right)^{1 / 2}}{6 d \nu\left(1+4 \beta^{2}\right)}\left(8 \beta d-D d^{2}+3 D\right) .
$$

On the line (21), this expression must be replaced by

$$
f_{1,2}=\frac{-(2 \beta-D \epsilon) \pm\left((2 \beta-D \epsilon)^{2}+\frac{9 \delta d^{2} \mu\left(1+4 \beta^{2}\right)^{2}}{\left(3 \beta-2 D d-\beta d^{2}\right)\left(D d-\beta+\beta d^{2}\right)}\right)^{1 / 2}}{3 d \mu\left(1+4 \beta^{2}\right)}\left(3 \beta-2 D d-\beta d^{2}\right) .
$$

The soliton solution (24) exists for both signs in expression (6) for $d$. The range of existence in the $(\beta, \epsilon, \mu, \nu, D)$ parameter space is restricted by the relation [Eq. (4)], which is a consequence of ansatz (3). In addition, relation (23) must be satisfied, and one of $f_{1,2}$ must be positive. Taking into account these two conditions, the region of existence of these solutions can be obtained.

As an illustrative example, Fig. 4 shows different curves which delimit the region of allowed values of the parameters $(\beta, \epsilon)$, where the solution exists for given values of $\nu, \delta$, and $D=-1$. The solid lines are for $\delta=-0.1$, and the dashed ones for $\delta=0.1$. In Fig. 4(a), $\nu=0.1$, and no solution is then found for $d=d_{-}$. In Fig. 4(b), $\nu=-0.1$, and there are solutions for both values of $d$. The arrows indicate if the solutions exist above or below the corresponding curve.

As in the cubic case, we performed a detailed linear stability analysis of these solutions. The results of this study for $D=-1$ are summarized in Fig. 5. Here we considered the cases with $\beta=0.5, \nu= \pm 0.1$, and $\delta= \pm 0.1$. Other values of the parameters produced similar results. The figures show the growth rate of the most unstable perturbation associated with each solution given by Eq. (24). The continuous lines are for the cases when $\delta=-0.1$, and the dashed lines for $\delta=0.1$.

In Fig. 5(a), $\nu=-0.1$ and $d=d_{+}$, and both the $x$ and $y$ scales are linear. This figure shows that for positive $\delta$ the perturbation growth rate is exactly equal to $\delta$; that is, their instability has its origin solely in the instability of the uniform background $\psi=0$, while the pulse itself is stable. On the other hand, for negative $\delta$, the growth rate becomes closer to zero as we move to its smallest allowed value. This happens when $f_{1}$ becomes close to $f_{2}$. The solution then has an amplitude profile with a flat top (see Sec. IV G). This behavior resembles that observed for the case of anomalous dispersion.

The curves shown in Fig. 5(b) are similar to that obtained for the general solution of the cubic CGLE. This can be expected, as this solution can be obtained from that of the quintic one when we make $\nu \rightarrow 0^{+}$. As in the previous case, for positive $\delta$ the growth rate is equal to $\delta$ almost in the whole interval, that is, its instability has its origin in the uniform background.

Figure 5(c) shows that the solutions obtained by taking $d=d_{-}$are, in general, much more unstable than those obtained for $d=d_{+}$. Again, for positive $\delta$, we obtain an interval of values of $\epsilon$ where the growth rate is equal to $\delta$. Note that in Figs. 5(a) and 5(b) we used a logarithmic scale in the $y$ axis. As commented upon in Fig. 4(a), no solution exists for positive $\nu$ when $d=d_{-}$.
The general conclusion from the above stability analysis is that, although an exact solution (24) to the quintic CGLE can be found when a specific relation between the parameters [Eq. (4)] is satisfied, all of them are unstable. An exception appears in the vicinity of the boundary that separates pulses from pairs of fronts. The perturbation growth rate of these soliton solutions falls to zero when we tend to this limit. These stable solutions have a flat top. They exist and are stable for both values of the dispersion parameter.

\section{Singularity at $\boldsymbol{\nu \rightarrow 0 ^ { - }}$}

When $\nu$ is negative, one of the solutions has a singularity at $\nu \rightarrow 0^{-}$. The value $(2 \beta-D \epsilon) / d$ must be positive and finite. Then $f_{2}$ has the limit $\left[3 \delta d\left(1+4 \beta^{2}\right)\right] /$ $\left[2\left(D d-\beta+\beta d^{2}\right)(2 \beta-D \epsilon)^{2}\right]$, and $f_{1}$ goes to infinity as $\left[-(\epsilon+2 \beta)\left(8 \beta d+d^{2}-3\right)\right] /\left[3 d \nu\left(1+4 \beta^{2}\right)\right]$, and so the soliton amplitude goes to infinity. The singularity does not occur when $\nu \rightarrow 0^{+}$. The second solution in the limit $\nu \rightarrow 0^{+}$coincides with solution (9), which applies in the case of the cubic CGLE. Clearly, this singularity is trivial and is not related to any solution.

\section{Solution with arbitrary amplitude}

Another singularity appears when $\delta=0$ and $D d$ $-\beta+\beta d^{2}=0$. This last occurs on the same line $S$ in the $(\beta, \epsilon)$ plane as in the cubic case [see Eq. (11)]. Then, a class of solutions with arbitrary amplitude exists,

$$
f(t)=\frac{3 d\left(1+4 \beta^{2}\right) P}{(2 \beta-D \epsilon)+S \cosh (2 \sqrt{P} t)},
$$

where $P$ is an arbitrary positive parameter and

$$
S=\left((2 \beta-D \epsilon)^{2}+\frac{18 d^{2} \nu\left(1+4 \beta^{2}\right)^{2}}{\left(8 \beta d-D d^{2}+3 D\right)} P\right)^{1 / 2} .
$$

The values $d$ and $\omega$ are given by

$$
\begin{aligned}
& d=\frac{\sqrt{1+4 \beta^{2}}-D}{2 \beta}, \\
& \omega=-d \frac{1+4 \beta^{2}}{2 \beta} P .
\end{aligned}
$$



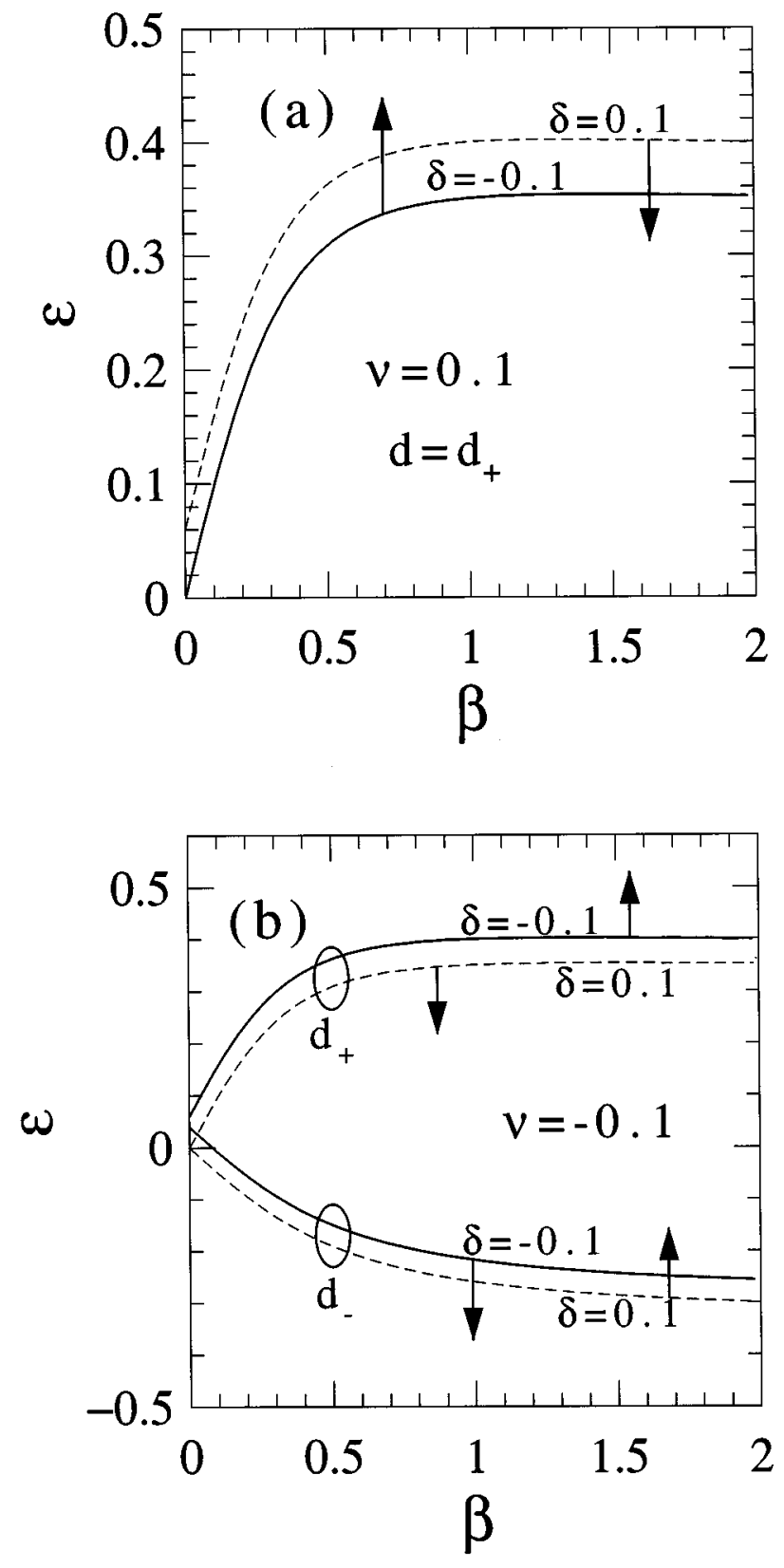

FIG. 4. Curves delimiting the regions on the $(\beta, \epsilon)$ plane where the stationary solution given by Eq. (27) exists. The continuous lines are for $\delta=-0.1$ and the dashed lines for $\delta=0.1$. In (a), $\nu=0.1$, and no solutions exist when taking $d=d_{-}$. (b) $\nu=0.1$. The arrows indicate if the solution exists above or below the curves.

As for the cubic case, we found, from numerical simulations, that in the normal dispersion regime $(D=-1)$ this class of solutions is unstable at any point of the special line $S$ and for any $P$ in Eq. (28).

\section{E. Flat-top solitons}

The soliton (24) becomes wider and flatter as the two positive $f_{1,2}$ approach each other. When $f_{1}=f_{2}$, the soliton splits into two fronts with zero velocity. Each of them can be written in the form (we ignore the translations along $t$ )

$$
f(t)=\frac{f_{1}}{1+\exp \left( \pm \alpha f_{1} t\right)},
$$
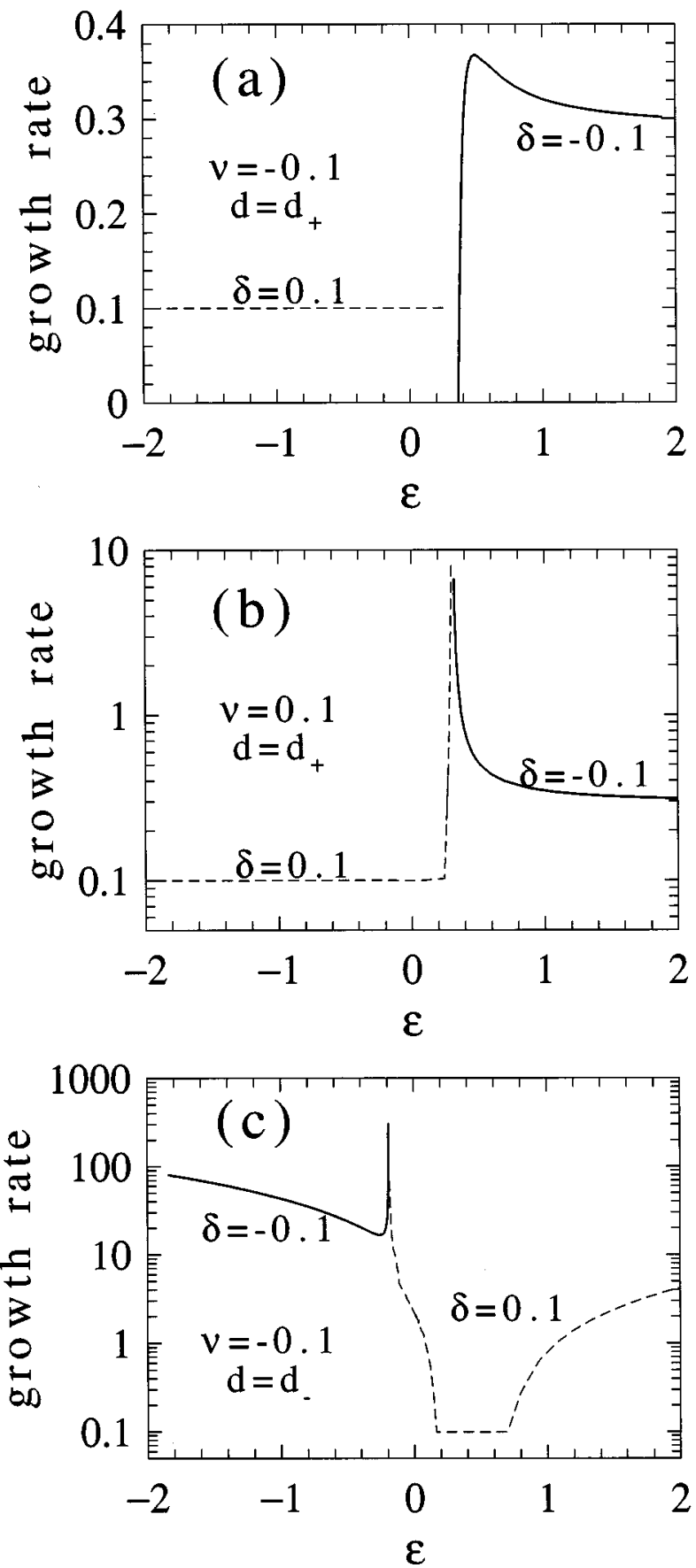

FIG. 5. The perturbation growth rate of the fixed amplitude solutions for $\beta=0.5$ and $\delta= \pm 0.1$. In (a) $\nu=-0.1$, and $d=d_{+}$. In (b), $\nu=0.1$, and $d=d_{+}$. In (c), $\nu=-0.1$, and $d=d_{-}$. The continuous lines are for $\delta=-0.1$, and the dashed lines for $\delta=0.1$.

where

$$
f_{1}=\frac{(\epsilon-2 D \beta)\left(8 \beta d-D d^{2}+3 D\right)}{6 d \nu\left(1+4 \beta^{2}\right)},
$$

and the sign in Eq. (32) determines the orientation of the front. $f_{1}$ and $f_{2}$ become identical when:

$$
(2 \beta-D \epsilon)^{2}=-\frac{18 \delta \nu d^{2}\left(1+4 \beta^{2}\right)^{2}}{\left(D d-\beta+\beta d^{2}\right)\left(8 \beta d-D d^{2}+3 D\right)} .
$$


This condition involves all the parameters of the equation. Depending on $\delta$ and $\nu$, it can exist at any point of the plane $(\epsilon, \beta)$. The top of the soliton becomes flatter as the roots become closer to each other, and as indicated in Fig. 5(a) the solution becomes more and more stable.

If $f_{1}=f_{2}$ exactly, the width of the pulse goes to infinity, and the pulse decomposes into two fronts. Note that, in the region of nonzero intensity, the soliton phase $\phi(t)$ tends to a constant value exponentially. So, if we combine the two fronts, Eq. (32), with opposite orientations, to form a wide, rectangular pulse of finite width, the influence of each front on the other is exponentially small. In other words, the two fronts, Eq. (32), can join together without any domain boundary between them (cf. [46]).

Pulses and fronts have usually been considered as different solutions of the CGLE [45-47]. Our results show that they can be transformed into each other by changing the parameters of the system. Moreover, our results give, at least partly, the range of parameters where we can expect smooth transitions from solitons to fronts. Stable stationary flat-top pulses have been observed experimentally in binary fluid convection [5].

\section{F. Algebraic solution}

If $\delta=0$ and $(\beta, \epsilon)$ is not located on line (11), then $\omega=0$, and either $f_{1}$ or $f_{2}$ (say $f_{2}$ ) becomes zero. Then $f_{1}$ is

$$
f_{1}=\frac{(\epsilon-2 D \beta)\left(8 \beta d-D d^{2}+3 D\right)}{3 d \nu\left(1+4 \beta^{2}\right)} .
$$

Equation (22) can then be written in the form

$$
f^{\prime 2}+4 k_{0}\left[f-f_{1}\right] f^{3}=0
$$

where $k_{0}=2 \nu /\left(8 \beta d-D d^{2}+3 D\right)$. The solution to this equation is a Lorentz function,

$$
f(t)=\frac{f_{1}}{1+k_{0} f_{1}^{2} t^{2}}
$$

The values $f_{1}$ and $k_{0}$ must be positive, which restricts the allowed values of the coefficients of the equation for this solution to exist.

The algebraic soliton is unstable for the full range of the parameters where it exists. It represents a special, weakly localized limit of the solution with fixed amplitude (24). Note that algebraic solitons exist, and play an important role, in other integrable and nonintegrable systems, including the NLSE [49].

\section{G. Traveling pulses}

If $\beta=0$, then solitons with nonzero velocity (traveling solitons) become possible. These solutions can be obtained using a simple transformation, because, for $\beta=0$, Eq. (1) has an additional symmetry, namely, it is invariant relative to the Galilean transformation. As a result, traveling pulselike solutions can be obtained from zero-velocity ones using the transformation

$$
\psi^{\prime}(z, t)=\psi(z, t-D v z) \exp \left(i v t-i D \frac{v^{2}}{2} z\right)
$$

Hence we can use the fixed amplitude solution of Sec. IV $\mathrm{B}$, put $\beta=0$, and use transformation (38) to get the whole family of traveling pulses. Note that all the analysis of Sec. IV $B$ is valid in this case. The critical points in $\epsilon$ are the intersections of the special lines in Figs. 1 and 4 with the vertical axis, $\beta=0$. This last example completes the classification of possible pulselike analytic solutions for Eq. (1).

\section{REGIONS IN PARAMETER SPACE WHERE STABLE PULSES EXIST}

In this section we obtain numerically the values of the coefficients $(\delta, \beta, \epsilon, \mu, \nu)$ of the quintic CGLE (subspace of the parameter-space) for which stable pulses exist. We find stable pulses in a certain region of parameters, and compare it with the region of a lower dimensionality where the analytical solutions given by Eq. (24) exist.

From a practical point of view, the knowledge of the parameter space where we can have stable pulse propagation, is of crucial importance. In addition, one can expect unusual propagation dynamics around the boundaries of the region of existence of stable pulses. For the case of anomalous dispersion, we discovered the existence of composite pulses and moving pulses near the upper boundary [50]. However, in this paper we look only for plain pulses.

Let us first fix some limits in the parameter space in which to look for stable pulses. The parameter $\beta$ clearly must be non-negative, in order to stabilize the soliton in the frequency domain. The linear gain coefficient $\delta$ must be zero or negative to provide the stability of the background. We choose $\mu<0$ to stabilize the pulse against the collapse. The parameter $\nu$ can have either sign.

Taking these restrictions into account, we have numerically found stable solutions following the method described in Ref. [29]. Figure 6 shows three examples for $D=-1$ of the soliton solutions found by using the above-referenced method.

In the same way we were able to construct the whole region in the parameter space where a stable propagation of bounded solutions is possible. Figures 7(a) and 7(b) show the areas in the $(\beta, \epsilon)$ plane were soliton solutions were numerically found for $\nu=-0.1$ and -0.01 , respectively. In both cases $D=-1, \delta=-0.1$, and $\mu=-0.1$. The lower curve (dashed line) represents line $S$, and it is plotted to allow us to make some comparisons with the conclusions which we obtained concerning the analytic solutions. First of all, let us notice that the region of stable pulses is always above line $S$, and that the lower boundary of the stability region (solid line) is approximately parallel to line $S$. The distance between this lower boundary and line $S$ depends on $\delta, \mu$, and $\nu$. For small $\mu, \nu$, and $\delta$ this distance is small. For given values of $\nu$ and $\delta$ the filled regions become wider as $|\mu|$ increases, and its lower boundary becomes higher. For given values of $\nu$ and $\mu$ the lower boundary approaches line $S$ as $\delta$ goes to zero. We may expect that at zero $\delta$ line $S$ would be the onset of instability. We also notice that the region of stable pulses in the parameter space starts at finite values of 

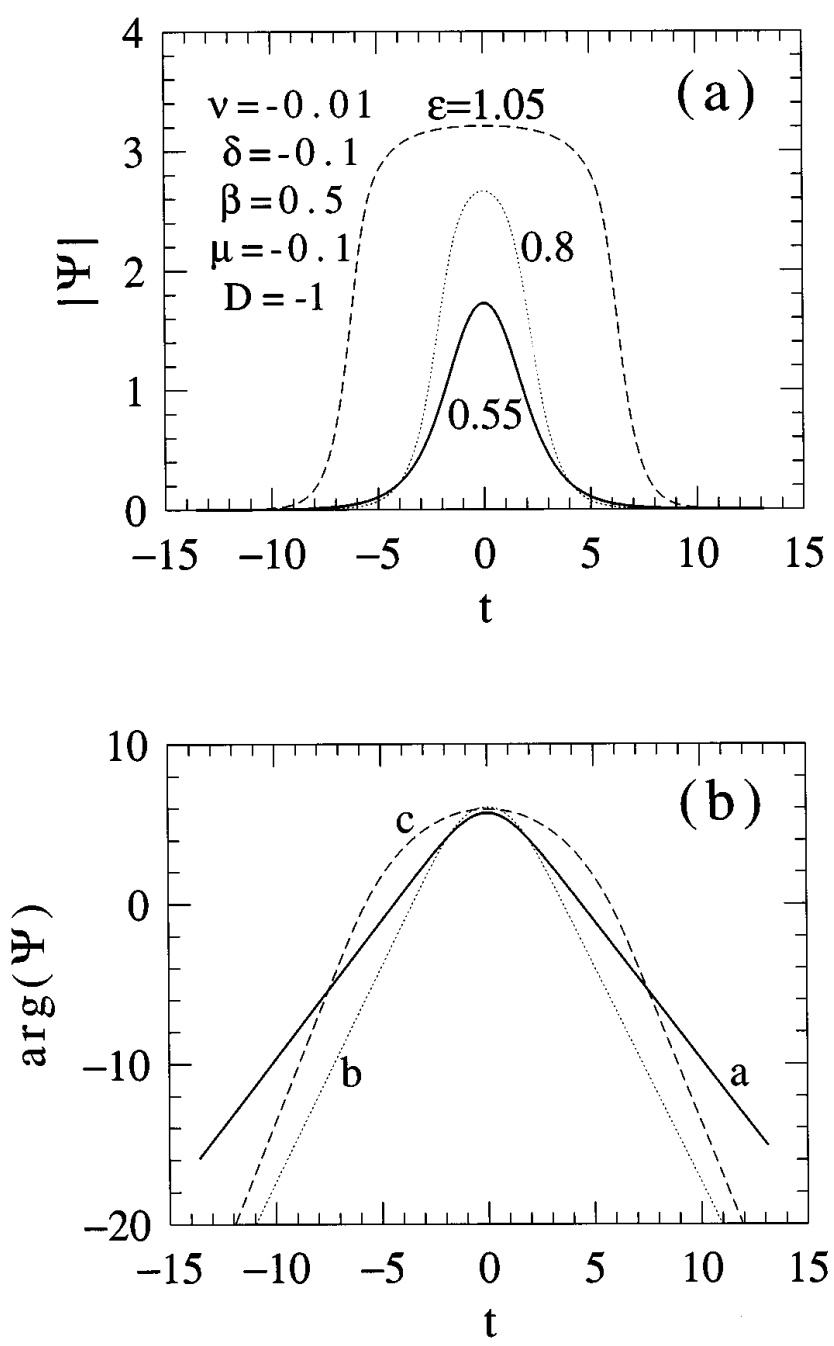

FIG. 6. Numerically found soliton solutions for (a) amplitude profile $|\psi|$. (b) Phase profile $\arg (\psi)$.

$\beta$. We suppose that stable pulses do not exist in the limit of $\beta \rightarrow 0$.

The upper boundary of the region of stable pulses in the $(\epsilon, \beta)$ plane corresponds to the transition from pulses to fronts [50]. Thus the solutions close to the upper boundary can be considered to be composed of two fronts. Examples of pulse solutions for the three different points of the shaded region designated in Fig. 7(b) by circles are shown in Fig. 7(c). The lower boundary of the region of stable pulses corresponds to the limit when the gain is not enough to compensate for the losses. Below this boundary all pulses decay. Three examples of stationary pulses for the parameters located close to the lower boundary are shown in Fig. 7(d) [points $d, e$, and $f$ in Fig. 7(b)].

We now consider other planes in the parameter space where we found stable pulselike solutions. Figure 8 shows the region of stable pulses in the plane $(\nu, \epsilon)$ for fixed values of $\mu, \delta$, and $\beta$ as written in the figure. The plot shows that the width of the stripe in Fig. 7 increases largely as $\nu$ increases. The dashed line in Fig. 8 shows where the exact analytical solutions are located for the same set of parameters. Interestingly enough, this line is also almost parallel to the upper border of the area of stable pulses, but located some distance from it. This shows that the analytical solu-
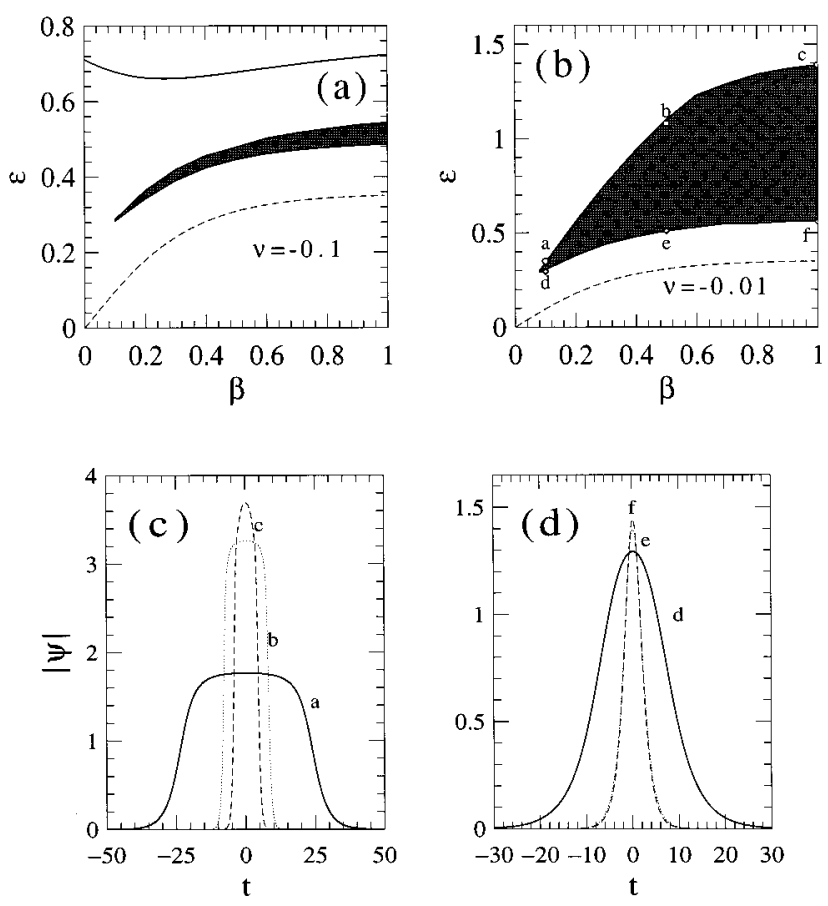

FIG. 7. (a) and (b) Regions in the $(\beta-\epsilon)$ plane where stable pulselike solutions are found for $D=-1, \mu=-0.1, \delta=-0.1$, and (a) $\nu=-0.1$ and (b) $\nu=-0.01$. These regions are located above line $S$ (dashed line). (c) and (d) Pulse profiles of six of these stable solutions located near the boundary in (b), as labeled therein.

tions are beyond that region and therefore, in total agreement with the stability analysis, unstable. White circles inside the filled region show the points corresponding to the numerical solutions of Fig. 6. These examples, together with those presented in Fig. 7(c), show that the upper boundary of the region of stable pulses corresponds to the transition from pulses to fronts.

It is also interesting to study how the region of stable pulses depends on $\delta$. Figure 9 shows this dependence for fixed values of $\mu, \nu$, and $\beta$. Specifically, $\nu=\mu=-0.1$ and $\beta=-0.5$. The filled area corresponds to $D=-1$, and the hatched area to $D=1$. As the linear loss decreases, the interval of allowed values of $\epsilon$ increases. The central value of this interval increases as $\delta$ increases. This means that the larger linear losses must be compensated (provided that other parameters are constant) for by the increased third-order nonlinear gain. For the above values of $\nu, \mu$, and $\beta$, Eq. (4) gives $\epsilon=1$ for $D=1$ and $\epsilon=0.677$ for $D=-1$. This value is, as expected, above the filled or hatched region. Figure 9 also shows that for the same set of parameters the region of existence of stable solutions is larger in the case of anomalous dispersion than in the case of normal dispersion. This difference decreases as the nonconservative parameters become greater.

Figure 10(a) shows the region of stable pulses in the plane $(\mu, \epsilon)$ for $D=-1$, and fixed values of $\nu, \delta$, and $\beta$ written in the figure. As $|\mu|$ increases, the interval of allowed values of $\epsilon$ becomes wider, and its central value larger. This last observation can also be expected, as it indicates that larger fifth-order nonlinear losses must be compensated for by the increasing third-order nonlinear gain. The width of the stripe 


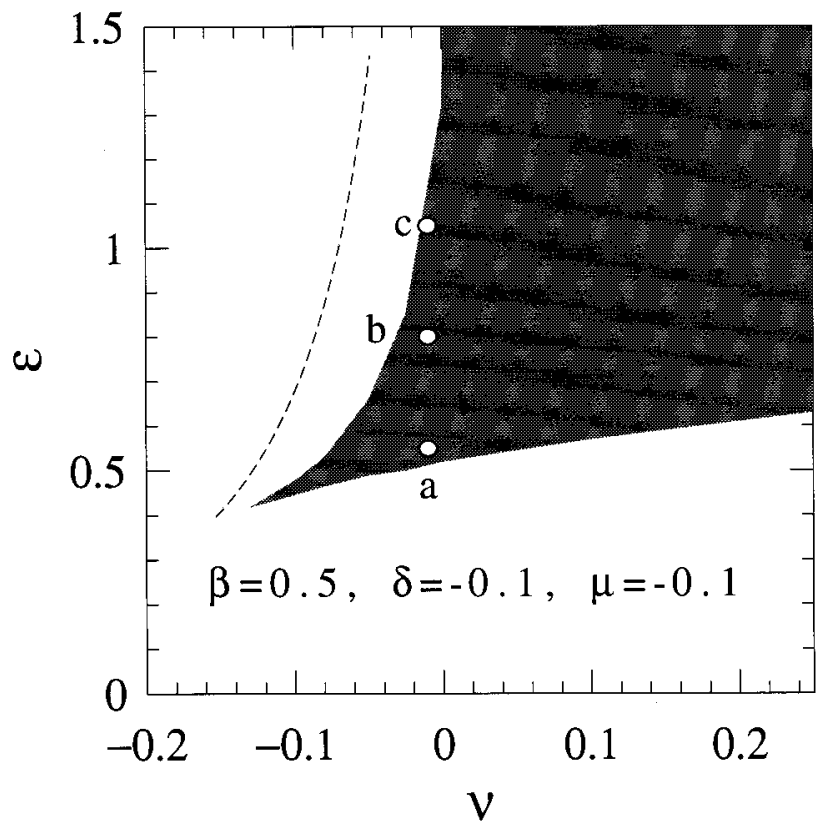

FIG. 8. Region in the plane $(\nu, \epsilon)$ where stable pulses are possible for $D=-1, \beta=0.5$, and $\mu=\delta=-0.1$. The dashed line represents the points where the analytical solution given by Eq. (24) exists. The open circles show the location of the stable solutions represented in Fig. 6.

becomes infinitesimally small at $\mu \approx-0.06$. The dashed line represents the points where the exact analytical solutions are located for the chosen values of $(\delta, \nu, \beta)$ $=(-0.1,-0.1,0.5)$. Again, it can be seen that they are out of the region of stable pulses. However, in this case the distance between the region of stable pulses and the exact analytical

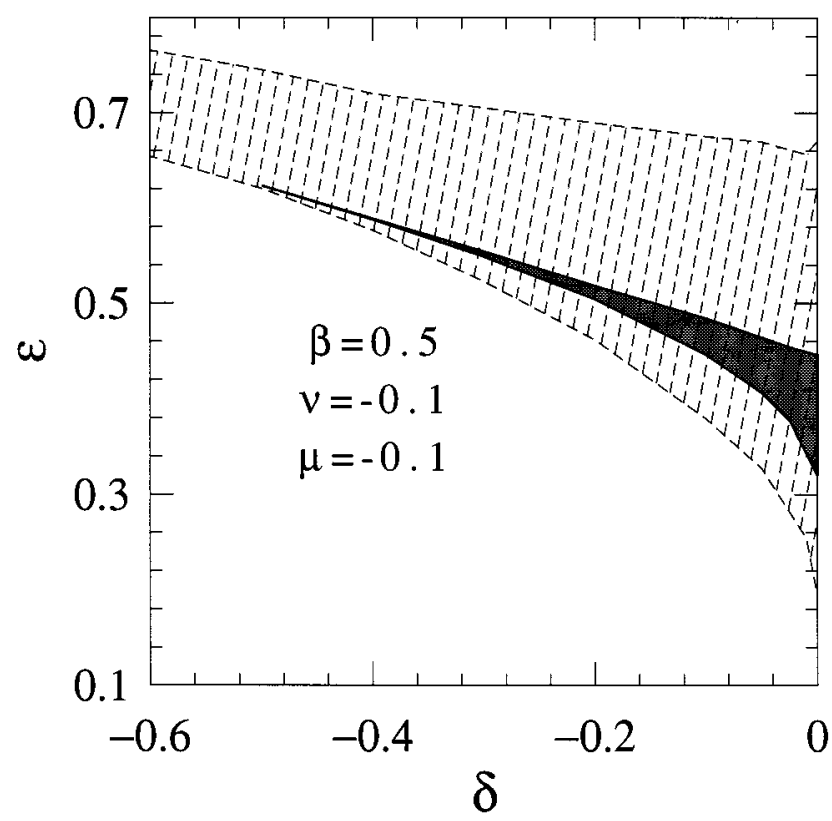

FIG. 9. Region in the plane $(\delta, \epsilon)$ where stable pulses are possible. $\beta=0.5$, and $\nu=\mu=-0.1$. The filled area is for $D=-1$, and the hatched region for $D=1$. For these parameters, the analytical solution exists at $\epsilon=1$ for $D=1$, and at $\epsilon=0.677$ for $D=-1$.
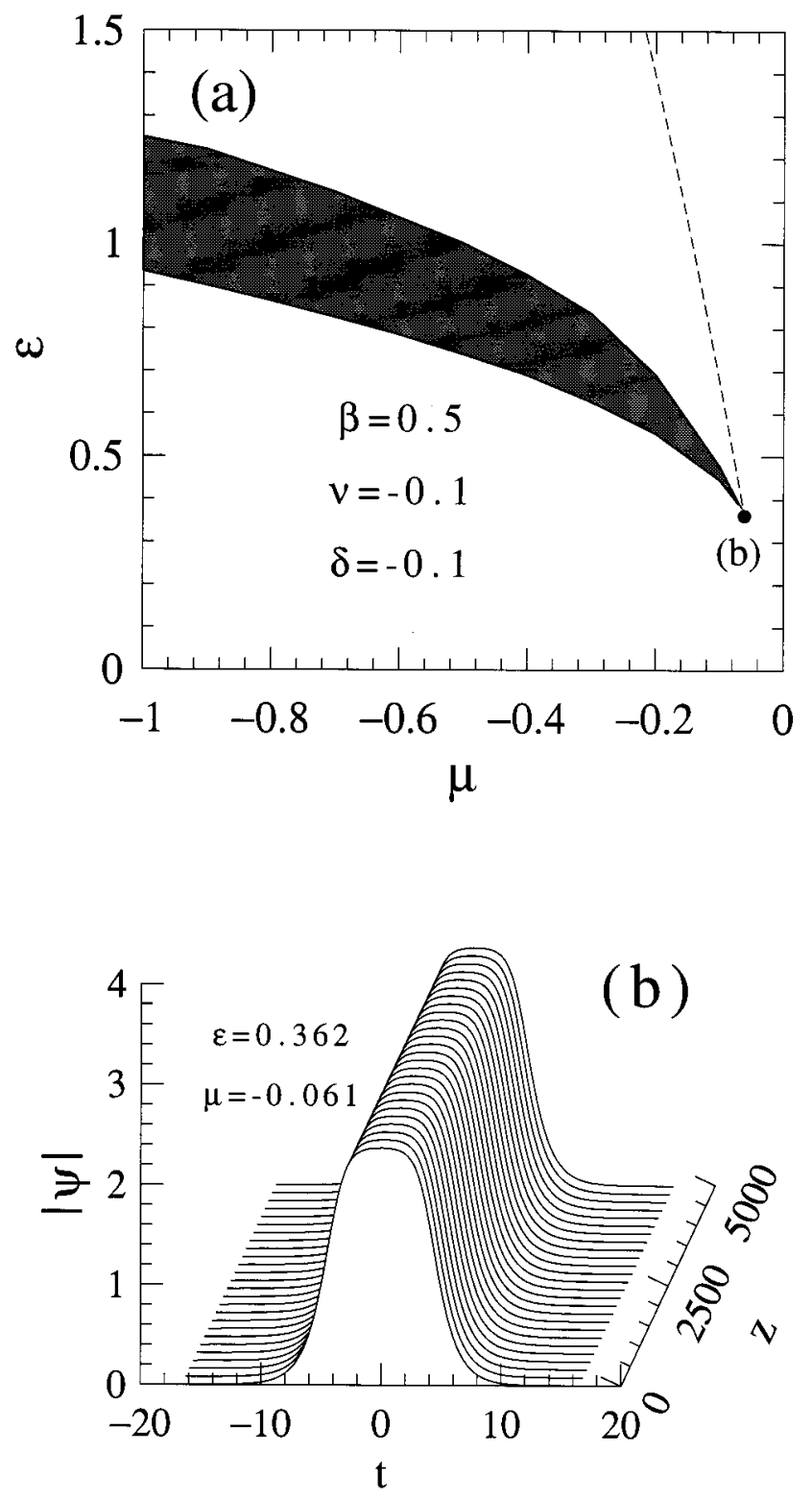

FIG. 10. (a) Region in the plane $(\mu, \epsilon)$ where stable pulses are found. $D=-1, \beta=0.5$, and $\nu=\delta=-0.1$. The dashed line represents the points where the analytical solution exists. (b) Stable propagation of the analytical solution (flat top soliton), for the values of the parameters shown in (a) by a filled circle.

solutions increases with $|\mu|$, and goes to zero at $\mu \rightarrow-0.04$. The instability growth rate of the corresponding analytical solution for this value of $\mu$ becomes neglectable. As expected from the stability analysis, this solution corresponds to a flat-top soliton. The example of propagation of the soliton at this point is shown in Fig. 10(b).

The imaginary part of the quintic term is essential for the existence of stable pulses. From Fig. 8, it follows that stable solutions exist for $\nu=0$. This is also true for positive $D$. On the other hand, Fig. 9 indicates that $\mu$ must have a finite negative value in order to stabilize the solutions. This minimal value depends on the values of other parameters. For $\nu=0$, and typical values for $(\beta, \delta)=(0.5,-0.1)$, we calculated the interval of values of $\epsilon$ at small $|\mu|$ where we can find stable solutions. The results are shown in Fig. 11(a). No 


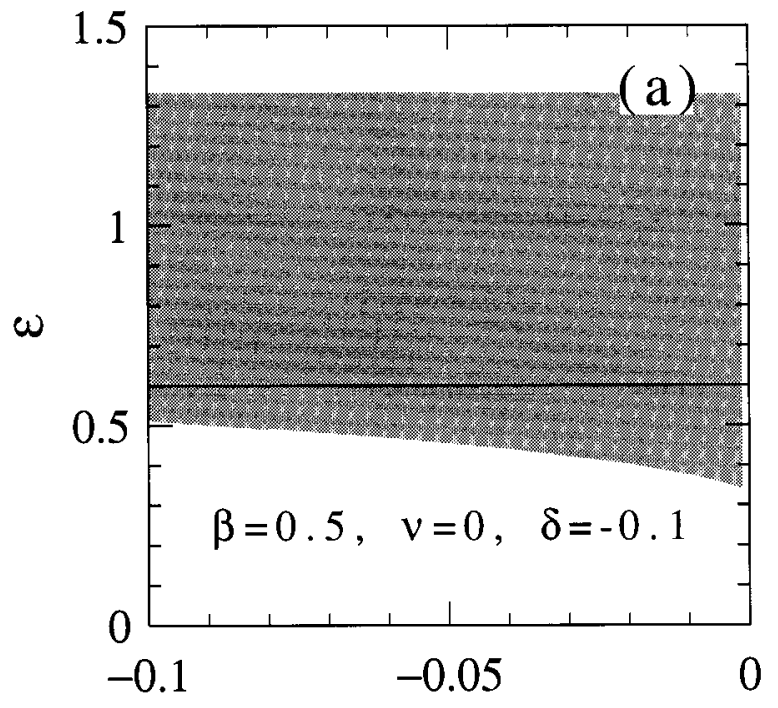

$\mu$

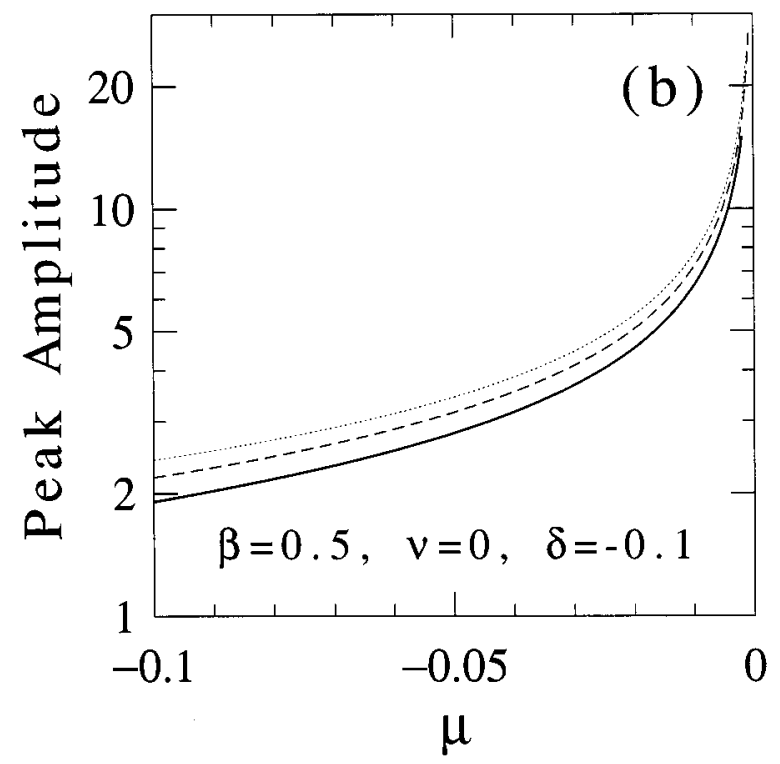

FIG. 11. (a) Region in the plane $(\mu, \epsilon)$ where stable pulses are found. $D=-1, \beta=0.5, \nu=0$, and $\delta=-0.1$. (b) Peak amplitude of the stable solution for the following parameters: $\beta=0.5, \nu=0$, $\delta=-0.1$, and $\epsilon=0.6$. The continuous line is for $D=-1$, the dashed line is for $D=1$, and the dotted line represents the cw solution.

solution is found if $\mu$ is exactly equal to zero, but there are solutions in a given interval of values of $\epsilon$ for any value of negative $\mu$, no matter how small its modulus is. The peak amplitude of the pulse diverges as $\mu$ tends to $0^{-}$. This is shown in Fig. 11(b), where we have represented the peak amplitude for the solutions obtained when $\epsilon=0.6$ [horizontal line in Fig. 11(a)]. The continuous line is for $D=-1$, the dashed line is for $D=1$, and the dotted line corresponds to the cw case, i.e., the amplitude obtained from the solution of the equation

$$
\delta+\epsilon\left|A_{\mathrm{CW}}\right|^{2}+\mu\left|A_{\mathrm{CW}}\right|^{4}=0 .
$$
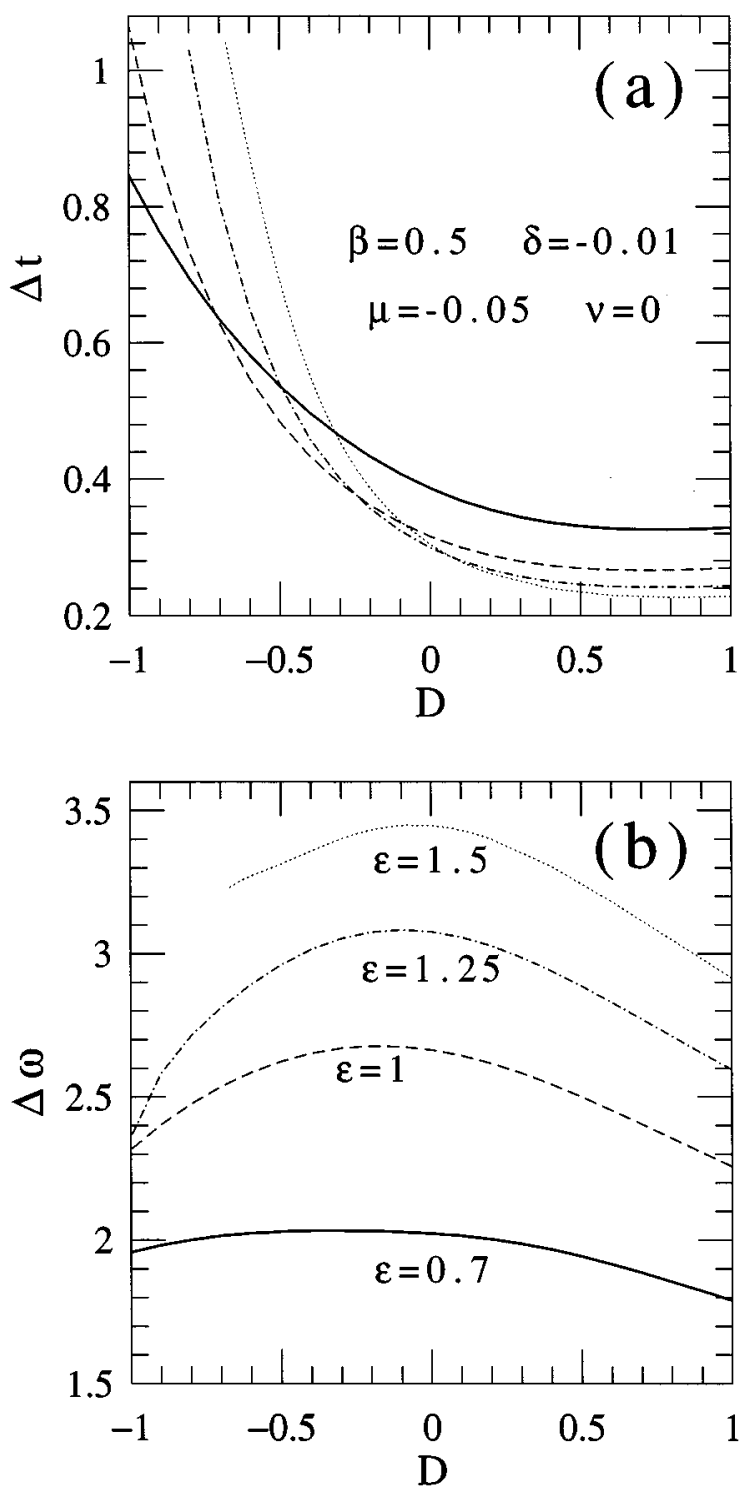

FIG. 12. Dependence on the dispersion coefficient $D$ of (a) the temporal width $\Delta t$, and (b) the spectral width $\Delta \omega$ of the stable pulses, for $\beta=0.5, \delta=-0.01, \mu=-0.05$, and $\nu=0$, for different values of the nonlinear gain coefficient $\epsilon$.

\section{ROLE OF GROUP-VELOCITY DISPERSION}

In order to establish a direct connection with experimental observations in physical systems that are describable in terms of the CGLE (e.g., a fiber laser [32,33]), in this section we present an example of the temporal and spectral characterization of the stable pulse solutions of Eq. (1). Such solutions (say, $\psi_{s}$ ) are obtained by means of the direct numerical integration of Eq. (1): we evaluate the pulse parameters after that the numerically propagated pulse has relaxed its temporal and spectral width to its asymptotic values [this typically requires a propagation over a distance $z \simeq 50 \mathrm{in} \mathrm{Eq.} \mathrm{(1)].}$

So far, in this work we considered the group-velocity dispersion $D$ as a fixed parameter, that is, we set $D=-1$ (1) for the normal (anomalous) dispersion case. However, in a fiber laser the average cavity dispersion can be easily tuned, for example, by changing the length of two fiber sections with opposite signs of the dispersion. Recent experiments with a "stretched-pulse laser' have shown, for example, that the 


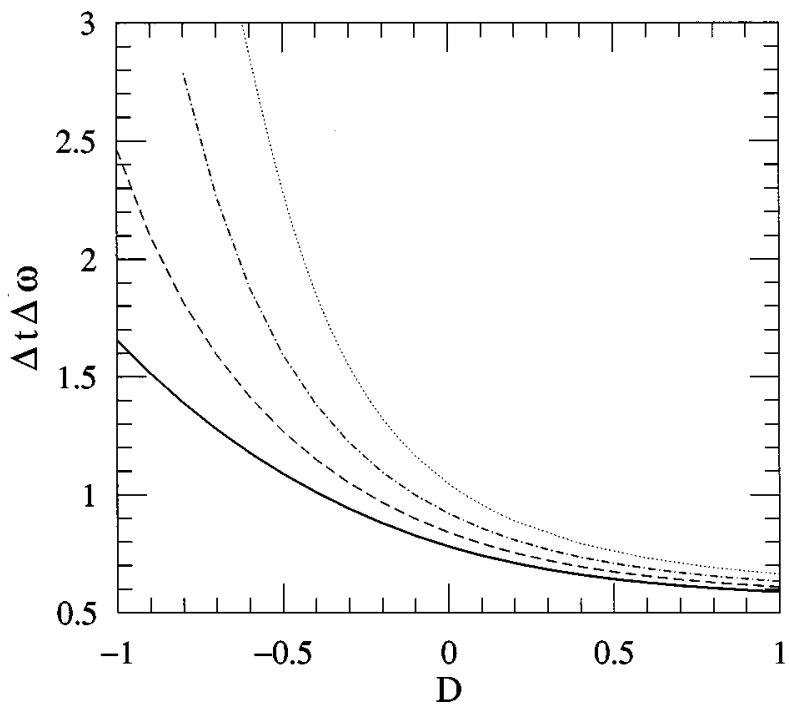

FIG. 13. Dependence on the dispersion coefficient $D$ of the time-bandwidth product $\Delta t \Delta \omega$ of the stable pulses, for the same parameters as in Fig. 12.

energy of the chirped pulses which are emitted from the laser grows considerably larger whenever the dispersion is continuously tuned from the anomalous to the normal regime [33].

In Figs. 12-14 we display the characteristics of the stable pulse solutions of the CGLE (1), as functions of the dispersion coefficient $D$, for different values of the parameter $\epsilon$. Here the nonlinear gain varies from $\epsilon=0.7$ (solid curves) to $\epsilon=1$ (dashed curve), $\epsilon=1.25$ (dot-dashed curve), and $\epsilon=1.5$ (dotted curve). For simplicity, we restricted our attention to the specific case where all other parameters in Eq. (1) are fixed, i.e., we set $\beta=0.5, \delta=-0.01, \mu=-0.05$, and $\nu=0$. We define the temporal width $\Delta t$ as

$$
\Delta t \equiv \sqrt{\left\langle t^{2}\right\rangle-\langle t\rangle^{2}}
$$

where

$$
\left\langle t^{n}\right\rangle \equiv \frac{\int t^{n}\left|\psi_{s}\right|^{2} d t}{\int\left|\psi_{s}\right|^{2} d t}, \quad n=1,2, \ldots
$$

and similarly for the spectral width $\Delta \omega$. Figure 12(a) shows that the pulse width $\Delta t$ is almost constant for positive $D$ 's, whereas it grows rapidly larger in the normal dispersion regime: pulses about five times longer are obtained when $D$ switches from $D=+1$ to $D=-1$. The increase of the nonlinear gain coefficient $\epsilon$ has opposite effects on the pulse width in the two dispersion regimes: larger nonlinear gains lead to longer (shorter) pulses in the case of normal (anomalous) dispersion, respectively. On the other hand, Fig. 12(b) shows that the spectral width of the stable solitary pulses reaches a maximum whenever the dispersion $D$ takes small negative values. Whereas the spectral width (hence the chirp) is reduced as the absolute value of the dispersion $D$ grows larger, irrespective of its sign. Moreover, Fig. 12(b) also shows that larger values of the nonlinear gain coefficient $\epsilon$
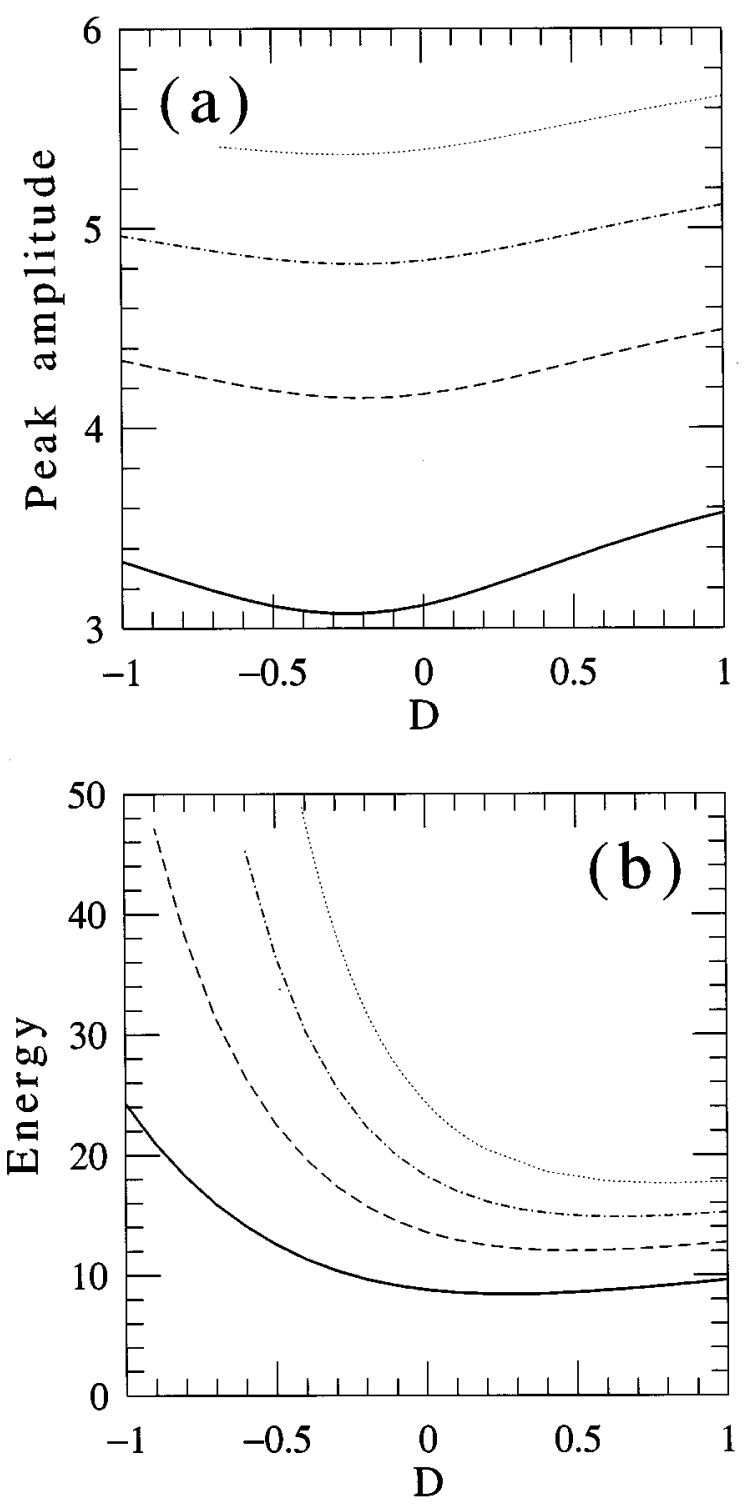

FIG. 14. Dependence on the dispersion coefficient $D$ of (a) the peak amplitude and (b) the energy of the stable pulses.

lead to a spectral broadening which is mostly due to an enhanced chirping rather than to temporal compression of the pulses [see Fig. 12(a)].

In fact, the contribution of chirping to the spectral width of the solitary wave solutions of Eq. (1) is well displayed by the plots of Fig. 13, where we show the variation with $D$ of the time-bandwidth product $\Delta t \Delta \omega$. As can be seen, in the anomalous dispersion regime $\Delta t \Delta \omega$ grows from 0.5 (0.6) up to 0.7 (1) for $\epsilon=0.7$ (1.5). In the normal dispersion regime $\Delta t \Delta \omega$ exhibits an exponential growth which reveals the occurrence of strong pulse chirping. Note that this chirp may be exploited in practice for achieving pulses that are strongly compressed in time, if, for example, the pulses from a fiber laser with an average normal dispersion are injected in a fiber lead with anomalous group-velocity dispersion [33].

Two other important characteristics of the stable pulse solutions of the CGLE are illustrated in Fig. 14, where we show the $D$ dependence of their peak amplitude [Fig. 14(a)] and energy $E \equiv \int\left|\psi_{s}\right|^{2} d t$ [Fig. 14(b)]. As can be seen, for a 
given value of the nonlinear gain parameter $\epsilon$, the peak amplitude is nearly independent of the group-velocity dispersion, whereas the amplitude grows monotonically with the absolute value of $\epsilon$. On the other hand, the pulse energy is almost constant with respect to $D$ for $D>0$, and it grows exponentially larger as soon as decreases below zero.

\section{DISCUSSION}

In the above analysis, we found pulselike solutions of the cubic and quintic CGLE for both anomalous and normal dispersion cases. All solutions are written in explicit form in terms of the parameters of the CGLE. Two different classes of solutions exist: solutions with fixed amplitude and solutions with arbitrary amplitude. The arbitrary-amplitude solitons exist on special lines in the parameter space where solutions with fixed amplitude become singular. However, it occurs that in the case of normal dispersion these solutions are always unstable, whereas they are stable for anomalous dispersion.

For the cubic case, our solutions cover all possible pulses, including solitons with fixed amplitude and the class of arbitrary amplitude solitons. In the quintic case, the solution can be explicitly written in a subspace of the full space of the coefficients of a lower dimensionality. This is a consequence of using ansatz (3). This subspace is described by Eq. (18). One of the ways to step out beyond the limitations of Eq. (18) would be the development of a perturbation theory using our set of solutions as unperturbed solutions.

The results of our work can be applied to different physical problems. These days, the most promising areas where the solutions of CGLE can give insight are optical telecommunications and laser physics. Consequently, here we discuss only the use of solutions in this important general area, leaving aside other possible applications.

The cubic CGLE is a good model for describing optical transmission systems with guiding filters $[8,9]$. The use of nonlinear gain $(\epsilon>0)$ in these systems allows the reduction or suppression of the growth of linear radiation $[12,14,40]$. The present results show, in particular, that stronger spectral filtering $(\beta \sim 1)$ than what has been considered before $(\beta \ll 1)$ can be used in these systems. In this case Eq. (11), derived here, gives the instability threshold. The propagation distance of "stable" pulses in the normal dispersion regime is limited by the instability of the background. Therefore, strictly speaking, all solutions of the cubic CGLE in the normal dispersion regime are unstable. As a consequence, in order to describe stable pulse formation in fiber laser systems properly, it is necessary to introduce higher-order nonlinear terms (or frequency sliding as discussed in Ref. [32]).

As a physical system which is described by the quintic CGLE one may consider, as we mentioned above, a soliton fiber laser with nonlinear polarization-dependent losses (which is equivalent to fast saturable absorption action $[1,12,19])$. In this case, the time-localized pulse is supported by the nonlinear gain, and loses energy due to three effects: spectral filtering, linear losses, and the quintic stabilizing term. However, even a small linear loss is enough to keep the background state stable. So a stable stationary soliton state may be formed as a result of the balance between nonlinear gain, spectral filtering, and the quintic stabilizing term. It is quite remarkable that, in the normal dispersion regime, the stable solutions of the cubic-quintic CGLE exhibit an important qualitative agreement with the experimental observation of a nearly exponential growth of the pulse energy with the absolute value of the average dispersion [33].

The knowledge of the regions of stability is vital for the proper operation of a fiber laser. Numerical results as those obtained in this work may provide a rough estimate of the characteristics of the pulses which are emitted from the laser as the various parameters (i.e., bandwidth of the intracavity filter, polarization controllers setting, average cavity dispersion) are adjusted.

\section{CONCLUSION}

In conclusion, we found pulselike solutions of both the cubic and quintic CGLE in a generalized form, which is valid for both the anomalous and the normal dispersion regimes. We studied their stability through a linear stability analysis, and found that they are in general unstable. For the quintic equation we found regions in parameter space where the pulselike solutions are stable. A comparison of the characteristics of the generated pulses as a function of the magnitude and sign of the group-velocity dispersion was also made. Finally, the connection of the present analysis with the available experimental results was discussed.

\section{ACKNOWLEDGMENTS}

The work of J.M.S.-C. was supported by the CICyT under Contract No. TIC95-0563-C05-03 and by the Comunidad de Madrid under Contract No. 06T/039/96. N.A. would like to thank the Australian Academy of Sciences for financial support.
[1] J. D. Moores, Opt. Commun. 96, 65 (1993).

[2] H. Haken, Synergetics (Berlin, Springer, 1983).

[3] P. K. Jakobsen, J. V. Moloney, A. C. Newell, and R. Indik, Phys. Rev. A 45, 8129 (1992).

[4] G. K. Harkness, W. J. Firth, J. B. Geddes, J. V. Moloney, and E. M. Wright, Phys. Rev. A 50, 4310 (1994).

[5] P. Kolodner, D. Bensimon, and C. M. Surko, Phys. Rev. Lett. 60, 1723 (1988).

[6] P. Kolodner, Phys. Rev. A 44, 6448 (1991); 44, 6466 (1991); 46, 6431 (1992); 46, 6452 (1992).
[7] R. Graham, in Fluctuations, Instabilities and Phase Transitions, edited by T. Riste (Springer, Berlin, 1975).

[8] A. Mecozzi, J. D. Moores, H. A. Haus, and Y. Lai, Opt. Lett. 16, 1841 (1991); J. Opt. Soc. Am. B 9, 1350 (1992).

[9] Y. Kodama and A. Hasegawa, Opt. Lett. 17, 31 (1992).

[10] L. F. Mollenauer, J. P. Gordon, and S. G. Evangelides, Opt. Lett. 17, 1575 (1992).

[11] L. F. Mollenauer, E. Lichtman, G. T. Harvey, M. J. Neubelt, and N. M. Nyman, Electron. Lett. 27, 792 (1992). 
[12] Y. Kodama, M. Romagnoli, and S. Wabnitz, Electron. Lett. 28, 1981 (1992).

[13] H. R. Brand and R. J. Deissler, Physica A 204, 87 (1994).

[14] M. Matsumoto, H. Ikeda, T. Uda, and A. Hasegawa, J. Lightwave Technol. 13, 658 (1995).

[15] V. V. Afanasjev, Opt. Lett. 18, 790 (1993).

[16] O. E. Martinez, R. L. Fork, and J. P. Gordon, J. Opt. Soc. Am. 2, 753 (1985).

[17] C.-J. Chen, P. K. A. Wai, and C. R. Menyuk, Opt. Lett. 19, 198 (1994); 20, 350 (1995).

[18] P. A. Belanger, J. Opt. Soc. Am. B 8, 2077 (1991).

[19] H. A. Haus, J. G. Fujimoto, and E. P. Ippen, J. Opt. Soc. Am. B 8, 2068 (1991).

[20] M. Hofer, M. E. Fernmann, F. Haberl, M. H. Ober, and A. J. Schmidt, Opt. Lett. 16, 502 (1991).

[21] M. Hofer, M. H. Ober, F. Haberl, and M. E. Fermann, IEEE J. Quantum Electron. 28, 720 (1992).

[22] K. Tamura, H. A. Haus, and E. P. Ippen, Electron. Lett. 28, 2226 (1992).

[23] V. J. Matsas, T. P. Newson, and M. N. Zervas, Opt. Commun. 92, 61 (1992).

[24] D. U. Noske, N. Pandit, and J. R. Taylor, Electron. Lett. 28, 2185 (1992).

[25] V. J. Matsas, D. J. Richardson, T. P. Newson, and D. N. Payne, Opt. Lett. 18, 358 (1993).

[26] H. A. Haus, E. P. Ippen, and K. Tamura, IEEE J. Quantum Electron. 30, 200 (1994).

[27] C. De Angelis, M. Santagiustina, and S. Wabnitz, Opt. Commun. 122, 23 (1995).

[28] N. N. Akhmediev, V. V. Afanasjev and J. M. Soto-Crespo, Phys. Rev. E 53, 1190 (1996); N. N. Akhmediev and V. V. Afanasjev, Phys. Rev. Lett. 75, 2320 (1995).

[29] J. M. Soto-Crespo, N. N. Akhmediev, and V. V. Afanasjev, J. Opt. Soc. Am. B 13, 1439 (1996).

[30] V. I. Karpman and E. M. Maslov, Zh. Éksp. Teor. Fiz. 73, 537 (1977) [Sov. Phys. JETP 48, 281 (1978)].

[31] J. Weiland, Y. H. Ichikawa, and H. Wilhelmsson, Phys. Scr. 17, 517 (1978).
[32] M. Romagnoli, S. Wabnitz, P. Franco, M. Midrio, L. Bossalini, and F. Fontana, J. Opt. Soc. Am. B 12, 938 (1995); M. Romagnoli, S. Wabnitz, P. Franco, M. Midrio, F. Fontana, and G. E. Town, ibid. 12, 72 (1995).

[33] H. A. Haus, K. Tamura, L. E. Nelson, and E. P. Ippen, IEEE J. Quantum Electron. 31, 591 (1995).

[34] N. R. Pereira and L. Stenflo, Phys. Fluids 20, 1733 (1977).

[35] P. A. Bélanger, L. Gagnon, and C. Paré, Opt. Lett. 14, 943 (1989); C. Paré, L. Gagnon, and P. A. Bélanger, Opt. Commun. 74, 228 (1989).

[36] V. V. Afanasjev and N. Akhmediev, Opt. Lett. 20, 1970 (1995).

[37] L. M. Hocking and K. Stewartson, Proc. R. Soc. London Ser. A 326, 289 (1972).

[38] K. Nozaki and N. Bekki, J. Phys. Soc. Jpn. 53, 1581 (1984).

[39] R. Conte and M. Musette, Physica D 69, 1 (1993).

[40] V. V. Afanasjev, Opt. Lett. 20, 704 (1995).

[41] B. A. Malomed, Physica D 29, 155 (1987).

[42] V. Hakim, P. Jakobsen, and Y. Pomeau, Europhys. Lett. 11, 19 (1990).

[43] S. Fauve and O. Thual, Phys. Rev. Lett. 64, 282 (1990); O. Thual and S. Fauve, J. Phys. (Paris) 49, 1829 (1988).

[44] H. R. Brand and R. J. Deisler, Phys. Rev. Lett. 63, 2801 (1989).

[45] W. van Saarloos and P. C. Hohenberg, Phys. Rev. Lett. 64, 749 (1990).

[46] W. van Saarloos and P. C. Hohenberg, Physica D 56, 303 (1992).

[47] P. Marcq, H. Chaté, and R. Conte, Physica D 73, 305 (1994).

[48] N. N. Akhmediev, V. I. Korneev, and Yu. V. Kuz'menko, Zh. Éksp. Teor. Fiz. 88, 107 (1985) [Sov. Phys. JETP 61, 62 (1985)].

[49] N. N. Akhmediev, V. M. Eleonskii, and N. E. Kulagin, translation of Theor. Math. Phys. (USSR) 72, 809 (1987) [note that ordinary differential equation (ODE) (22) is a special case of the more general ODE (15) of this reference, the general solution to which is given by Eq. (24) of this reference].

[50] V. V. Afanasjev, N. Akhmediev, and J. M. Soto-Crespo, Phys. Rev. E 53, 1931 (1996). 